\title{
Enhanced sensitivity of laforin- and malin-deficient mice to the convulsant agent pentylenetetrazole
}

\section{Ana M. García-Cabrero ${ }^{1,2}$, Gentzane Sánchez-Elexpuru ${ }^{1,2}$, José M. Serratosa ${ }^{1,2 *}$ and Marina P. Sánchez ${ }^{1,2 *}$}

' Laboratory of Neurology, IIS-Fundación Jiménez Díaz, Universidad Autónoma de Madrid, Madrid, Spain

${ }^{2}$ Centro de Investigación Biomédica en Red de Enfermedades Raras, Madrid, Spain

\section{Edited by:}

Jesus Avila, Centro de Biología

Molecular Severo Ochoa Consejo

Superior de Investigaciones

Científicas-Universidad Autónoma

de Madrid, Spain

Reviewed by:

Matthew S. Gentry, University of

Kentucky, USA

Tobias Engel, Royal College of

Surgeons, Ireland

\section{*Correspondence:}

José M. Serratosa and Marina P.

Sánchez, Laboratory of Neurology,

IIS-Fundación Jiménez Díaz,

Universidad Autónoma de Madrid,

Av. Reyes Católicos 2,

28040 Madrid, Spain

e-mail: jmserratosa@fjd.es;

msanchezg@fjd.es
Lafora disease is a rare form of inherited progressive myoclonus epilepsy caused by mutations in the EPM2A gene encoding laforin, or in the EPM2B gene, which encodes malin. It is characterized by the presence of polyglucosan inclusion bodies (Lafora bodies) in brain and other tissues. Genetically engineered mice lacking expression of either the laforin $\left(E p m 2 a^{-I-}\right)$ or malin $\left(E p m 2 b^{-/-}\right)$genes display a number of neurological and behavioral abnormalities that resemble those found in patients suffering from Lafora disease; of these, both Epm2a $a^{-1-}$ and Epm2 $b^{-1-}$ mice have shown altered motor activity, impaired motor coordination, episodic memory deficits, and different degrees of spontaneous epileptic activity. In this study, we analyze the sensitivity of $E p m 2 a^{-/-}$and Epm2 $b^{-1-}$ mice to the convulsant drug pentylenetetrazol (PTZ), an antagonist of the $\gamma$-aminobutyric acid type $A\left(G A B A_{A}\right)$ receptor, commonly used to induce epileptic tonic-clonic seizures in laboratory animals. PTZ-induced epileptic activity, including myoclonic jerks and tonic-clonic seizures, was analyzed in 2 age groups of mice comprising representative samples of young adult and aged mice, after administration of PTZ at sub-convulsive and convulsive doses. Epm2a ${ }^{-1-}$ and Epm $2 b^{-1-}$ mice showed a lower convulsive threshold after PTZ injections at sub-convulsive doses. A lower convulsive threshold and shorter latencies to develop epileptic seizures were observed after PTZ injections at convulsive doses. Different patterns of generalized seizures and of

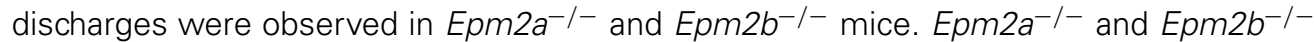
mice present an increased sensitivity to the convulsant agent PTZ that may reflect different degrees of increased $\mathrm{GABA}_{A}$ receptor-mediated hyperexcitability.

Keywords: Lafora disease, epilepsy, Epm2a-/- and Epm2b-/- mice, PTZ, seizure threshold

\section{INTRODUCTION}

Lafora disease (LD) is a rare form of inherited progressive myoclonus epilepsy (OMIM\#254780; ORPHA501). Recessive mutations in either the EPM2A gene encoding a dual-specificity phosphatase known as laforin (OMIM 607566) (Minassian et al., 1998; Serratosa et al., 1999) or in the EPM2B/NHLRC1 gene encoding malin (OMIM 608072), an E3 ubiquitin ligase (Chan et al., 2003; Gentry et al., 2005; Gomez-Abad et al., 2005; Singh et al., 2005, 2006) are responsible for the disease, although the existence of a third, minor locus has also been postulated (Chan et al., 2004).

Patients with LD develop progressive myoclonus epilepsy that usually starts in adolescence and includes absence, visual, myoclonic, and tonic-clonic seizures accompanied by rapid neurologic degeneration, including ataxia, dementia, dysarthria, amaurosis, respiratory failure, and a final vegetative state and death, usually within 10 years of onset (Lafora, 1911; Van Heycop Ten Ham, 1974; Berkovic et al., 1986, 1991; Roger et al., 1992; Acharya et al., 1995). The principal pathologic feature of LD is the presence of periodic acid-Schiff (PAS)-positive intracellular inclusions of polyglucosans (Lafora bodies) located mainly in the brain, the skeletal muscle, the heart, and the liver (Lafora, 1911; Lafora and Glueck, 1911; Harriman et al., 1955; Yokoi et al., 1968; Sakai et al., 1970; Carpenter and Karpati, 1981; Berkovic et al., 1986). Currently, no preventive or curative therapies exist for LD.

Different mouse models have been generated by disrupting either the Epm2a (Ganesh et al., 2002) or the Epm2b gene (Depaoli-Roach et al., 2010; Turnbull et al., 2010; Valles-Ortega et al., 2011; Criado et al., 2012). Of these, the Epm2a $a^{-/-}$mouse line generated by Ganesh et al. (2002) displays many of the neurological and behavioral abnormalities found in LD patients, including neuronal degeneration and the development of Lafora bodies in different organs including heart, liver, muscle, and a variety of brain regions such as hippocampus, cerebral cortex, thalamus, cerebellum, and brainstem (Ganesh et al., 2002). In addition, the Epm $2 b^{-/-}$mouse line previously generated by our group (Criado et al., 2012) also shows substantial neurological abnormalities that correlated with the detection of Lafora bodies in the same brain regions as those of the Epm2a $a^{-/-}$mouse line (Criado et al., 2012). Of those neurological abnormalities, both laforin-deficient and malin-deficient mouse lines showed altered 
motor activity, impaired motor coordination, episodic memory deficits, and different degrees of spontaneous epileptic activity, such as spontaneous single spikes, polyspikes and spike-wave and polyspike-wave complexes correlating with myoclonic jerks (Ganesh et al., 2002; Criado et al., 2012; Garcia-Cabrero et al., 2012). Epm $2 a^{-/-}$and Epm $2 b^{-/-}$mice had spontaneous tonicclonic seizures, although the Epm $2 b^{-/-}$mice did not display EEG correlates (Garcia-Cabrero et al., 2012).

Here we analyze the responses of both $E p m 2 a^{-/-}$and Epm $2 b^{-/-}$mice to the administration of the epileptogenic agent pentylenetetrazole (PTZ). PTZ is widely used for the study of epileptiform activity in laboratory animals (Vernadakis and Woodbury, 1969; Reinhard and Reinhard, 1977; Swinyard et al., 1989), and is also used routinely to test antiepileptic drugs (Swinyard et al., 1989). PTZ is an antagonist of the type A receptor of $\gamma$-aminobutyric acid $\left(\mathrm{GABA}_{\mathrm{A}}\right)$ (Stone, 1970). As with other $\mathrm{GABA}_{\mathrm{A}}$ antagonists, administration of low doses of PTZ can result in absence seizures in animal models (Snead et al., 2000), whereas higher doses result in generalized tonic-clonic seizures (Woodbury et al., 1980; Panagopoulos et al., 1998; Coimbra et al., 2001a,b; Erakovic et al., 2001; Eloqayli et al., 2003). In this study, we used PTZ at both sub-convulsive and convulsive doses to analyze seizure thresholds in an attempt to elucidate the molecular mechanisms underlying altered neuronal excitability in Epm $2 a^{-/-}$and Epm $2 b^{-/-}$mice and to further evaluate novel therapeutic strategies to treat epileptic symptoms. PTZ induces seizure development shortly after injection (Pitkanen et al., 2006), with reversible effects and low toxicity at convulsive doses (Velisek, 2006), whereas other seizure inducing agents such as methionine sulfoximine or kainate (Dudek et al., 2006; Eid et al., 2008; Cloix and Hevor, 2009), induce development of seizures with long pre-convulsive periods of several hours, and others produce therapy-resistant seizures, e.g., strichnine (Löscher, 1997). This makes PTZ the most appropriate agent for seizure monitoring in our models.

\section{MATERIALS AND METHODS EXPERIMENTAL ANIMALS}

Generation of Epm $2 a^{-1-}$ mutant mice (Ganesh et al., 2002) was performed by targeted deletion of the fourth exon of the Epm2a gene (Minassian et al., 1998; Serratosa et al., 1999), which encodes the dual-specificity phosphatase domain that is critical for the function of laforin. Constitutive Epm $2 b^{-/-}$mutant mice were generated by targeting deletion of the unique exon encoding malin, as previously described (Criado et al., 2012). The genetic background of null-mouse lines used in the study was a mix of 129Sv:C57BL/6J (25\%:75\%). The mice were kept at the IIS-Fundación Jimenez Jiménez Díaz animal facility and were maintained on a 12:12-h light/dark cycle under constant temperature $\left(23^{\circ} \mathrm{C}\right)$ with free access to regular food and water ad libitum. Two groups of homozygous animals aged 4-8 and 9-23 months (representative samples of young adult and aged mice) were analyzed for each experiment. The experiments were conducted in accordance with the Declaration of Helsinki principles and the guidelines of the Institutional Animal Care and Use Committee, and were approved by the IIS-Fundación Jiménez Díaz ethical review board.

\section{HISTOLOGY AND IMMUNOSHISTOCHEMISTRY}

The animals were anesthetized with a mixture of medetomidine (Domitor, Pfizer, Espoo, Finland), ketamine (Ketolar, Pfizer, Espoo, Finland), and sterile water (1:1.5:1.5) and transcardially perfused with $4 \%$ phosphate-buffered paraformaldehyde. The animals' brains were removed, postfixed overnight at $4^{\circ} \mathrm{C}$, dehydrated in graded ethanol solutions, and embedded in paraffin. They were then sectioned in serial arrays of $7-\mu \mathrm{m}$-thick sections, deparaffinized at $60^{\circ} \mathrm{C}$, and stored at room temperature until use. For PAS staining, coronal sections were processed as previously described (Mitsuno et al., 1999), and counterstained with hematoxylin solution. For immunohistochemistry, contiguous PAS-stained sections were rehydrated and incubated in $1.5 \%$ ( $\mathrm{vol} / \mathrm{vol}$ ) $\mathrm{H}_{2} \mathrm{O}_{2}$ in methanol for $20 \mathrm{~min}$ at room temperature to inactivate endogenous peroxidase. Sections were then placed in blocking buffer for $60 \mathrm{~min}$ at room temperature and incubated for 3 days at $4^{\circ} \mathrm{C}$ with anti-ubiquitin antibody (Dako, Glostrup, Denmark). Subsequently, the sections were treated with anti-rabbit biotinylated secondary antibody and stained using the Vectastain ABC kit (Vector Laboratories, Burlingame, CA, USA). Immunoreactivity was developed with diaminobenzidine (Dako, Glostrup, Denmark), and sections were counterstained with hematoxylin solution, dehydrated, and covered with DePeX (SERVA Electrophoresis GmbH, Heidelberg, Germany).

\section{PTZ TREATMENT}

PTZ (Sigma Chemicals, St. Louis, MO, USA) was dissolved in sterile water and injected intraperitoneally at different doses as a single injection. The doses administered were those known to be sub-convulsive $(30 \mathrm{mg} / \mathrm{kg}$ ) (Erakovic et al., 2001) and convulsive (50 mg/kg) (Erakovic et al., 2001; Eloqayli et al., 2003) for control mice. As described previously, a dose of $50 \mathrm{mg} / \mathrm{kg}$ induces seizures in $50 \%$ of control animals (Shitak et al., 2006). After drug administration, PTZ-induced myoclonic jerks and seizure monitoring was performed in 16-36 mice per group using visual blinded observation over a period of $45 \mathrm{~min}$. The percentages of mice displaying myoclonic jerks and generalized tonic-clonic seizures were recorded in 3 groups of control, Epm $2 a^{-/-}$, and Epm $2 b^{-/-}$ mice at 4-8 and 9-23 months of age. Following injection of PTZ, mice displayed periods of immobility and overt evidence of convulsive activity which included hyperactivity, twitching, and hyperextension of the limbs that at times progresses to generalized tonic-clonic seizures. We recorded the percentages of PTZ injected mice that present both muscular jerks, which usually started within a few minutes of PTZ injection, and generalized tonic-clonic seizures. To assess for the presence of EEG correlates, twelve mice chosen at random as representative samples of these 3 groups at 4-8 and 9-23 months underwent surgery and analysis by video-EEG after PTZ injection at convulsive doses. EEG recordings of operated mice revealed the existence of isolated spikes and spike-waves that correlate with muscular jerks. Generalized tonic-clonic seizures with low-frequency $(3-6 \mathrm{~Hz})$ spike-wave correlates were also observed in EEGs. Latency-or time between injection and onset of seizures — and seizure length were also analyzed for those mice treated with $50 \mathrm{mg} / \mathrm{kg}$ PTZ. In addition to measuring epileptic activity, PTZ-induced lethality was also analyzed for each drug dose. 


\section{VIDEO-ELECTROENCEPHALOGRAPHIC (EEG) RECORDING}

Procedures for implantation of intracranial electrodes and video-EEG recording have been previously described (Criado et al., 2012; Garcia-Cabrero et al., 2012). Briefly, custom-made stainless-steel screw electrodes were fixed to the skulls of anesthetized animals under a mixture of medetomidine, ketamine and sterile water (1:1.5:1.5). Two monopolar epidural electrodes were implanted symmetrically over the frontal cortex in front of bregma, whereas 2 ground and reference electrodes were positioned posterior to lambda. After a 4-day recovery period, synchronized video-EEG activity was recorded using computerbased systems (Easy EEG 2.1, Cadwell, CA, USA and Natus Neurowork EEG, Natus Medical Inc., San Carlos, CA, USA) while the mice moved freely.

\section{STATISTICAL ANALYSIS}

Values are given as means \pm standard error of means (SEM) or percentages. Differences between groups were analyzed by two-tailed Student's $t$-test or Chi-square (Graph-Pad Prism 2.0). Statistical significance was considered to be reached at $* p<0.05$; ${ }^{* *} p<0.01 ;{ }^{* * *} p<0.001$.

\section{RESULTS}

\section{PRESENCE OF LAFORA BODIES IN Epm2a-/- AND Epm2b-/- MICE}

As in previous descriptions, Lafora inclusions were abundant in several brain regions of the Epm $2 a^{-/-}$and Epm $2 b^{-/-}$mice, including cerebral cortex, hippocampus, basal ganglia, thalamus, cerebellum, and brainstem (Criado et al., 2012; Garcia-Cabrero et al., 2012). Representative examples of neurons of Layers IV-V of the cortex exhibiting PAS-positive Lafora aggregates in cell bodies of Epm $2 a^{-/-}$and Epm $2 b^{-/-}$at 17 months of age are shown in Figures 1A,B, respectively. Intracellular inclusions immunostained for ubiquitin were also found in the same regions as PAS-positive inclusions (Figures 1C,D).

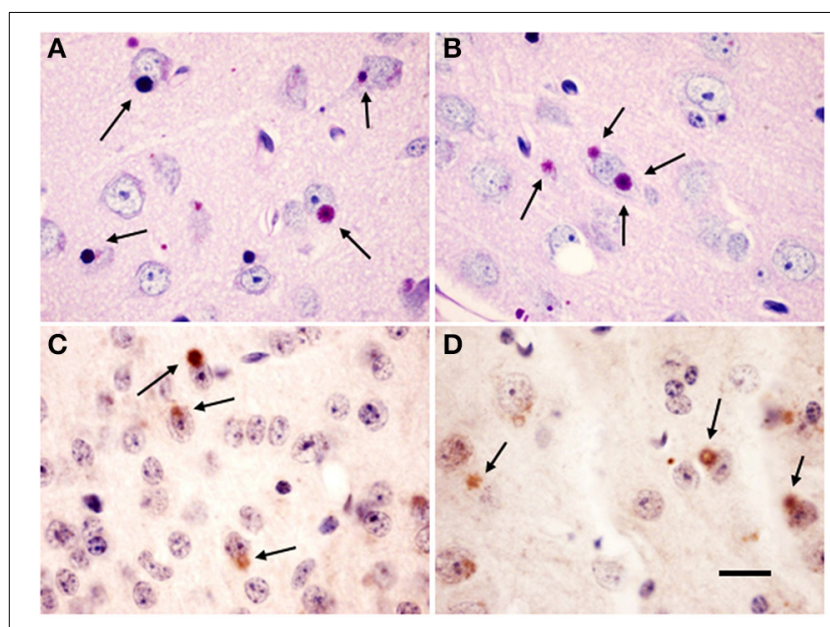

FIGURE 1 | Lafora bodies in Epm2a ${ }^{-/-}$and Epm2 ${ }^{-/-}$mice brains. Citoplasmic polyglucosan inclusions (arrows) positive for periodic acid-Shiff staining (PAS) $(\mathbf{A}, \mathbf{B})$ in layer $\mathrm{V}$ of the cerebral cortex, and immunostained for ubiquitin (C,D) in Layer IV (C) and IV-V (D) of cerebral cortex of Epm2a-1$(\mathbf{A}, \mathbf{C})$ and $E p m 2 b^{-/-}$(B,D) mice at 17 months of age. Scale bar $=20 \mu \mathrm{m}$.

\section{ANALYSIS OF PTZ-INDUCED MYOCLONUS, GENERALIZED TONIC-CLONIC SEIZURES, AND LETHALITY IN MICE LACKING LAFORIN OR MALIN}

Following injection of PTZ, control mice displayed periods of immobility and overt evidence of convulsive activity which included hyperactivity, twitching, and hyperextension of the limbs, later progressing to generalized tonic-clonic seizures. We established scores for the presence of both myoclonic jerks which usually started within a few minutes of PTZ injection, and tonicclonic seizures. EEG recordings of operated mice revealed the existence of isolated spikes and spike-wave correlates. At subconvulsive doses, the percentage of mutant mice showing myoclonic jerks was higher than that of control mice (Figure 2A). Thus, at this dose, $45 \%$ of Epm $2 a^{-/-}$and $50 \%$ of Epm $2 b^{-/-}$mice at $4-$ 8 months displayed myoclonic jerks, compared to only $7 \%$ of age-matched control mice. In the 9-to-23-month mice group, myoclonic jerks were present in $67 \%$ of Epm $2 a^{-/-}$and $75 \%$ of Epm $2 b^{-/-}$, compared to only $22 \%$ of control mice. When the induction of myoclonic jerks was analyzed at a higher dose of PTZ (50 mg/kg), no major differences were observed between controls and Epm $2 a^{-/-}$or Epm $2 b^{-/-}$mice (Figure 2B). These results indicate that a sub-convulsive dose of the epileptogenic drug PTZ induces the appearance of myoclonic jerks more easily in both young and old mice lacking the expression of either laforin or malin proteins, as compared to age-matched controls. Although both Epm $2 a^{-/-}$and Epm $2 b^{-/-}$mice trend to present more myoclonic jerks with age, no statistical differences were found between young and older mice. After administration of subconvulsive doses of PTZ in control, Epm $2 a^{-/-}$, or Epm $2 b^{-/-}$mice at 4-8 months of age, no generalized tonicclonic seizures were observed (Figure 2C). However, in the oldest group, a small proportion of mice displayed seizures, though no significant differences were recorded between con-

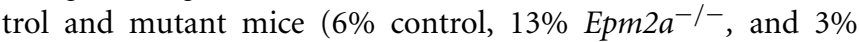
$E p m 2 b^{-/-}$) (Figure 2C). As expected, a convulsive PTZ dose at 4-8 months of age induced tonic-clonic seizures in 50\% of control mice (Figure 2D). Interestingly, this percentage was notably increased for both Epm $2 a^{-/-}(80 \%)$ and $E p m 2 b^{-/-}(78 \%)$ mice (Figure 2D). Similar results were obtained for Epm $2 a^{-/-}(78 \%)$ mice at 9-23 months, whereas no major differences between control and Epm $2 b^{-/-}$mice were noticed at this age (Figure 2D). Lethality thresholds were not affected in either Epm $2 a^{-/-}$or Epm $2 b^{-/-}$mice at any PTZ dose as compared to age-matched controls (data not shown).

\section{PTZ-INDUCED SEIZURE LATENCY AND LENGTH}

Latency, or the time elapsed between injection of a convulsive dose of PTZ (50 mg/kg) and tonic-clonic seizure appearance, was recorded for controls, Epm $2 a^{-/-}$, and Epm $2 b^{-/-}$mice. Mutant mice in both age ranges showed statistically significant reductions in seizure latency when compared to age-matched control mice (Figure 3A). Additionally, the length of PTZ-induced seizures in the Epm $2 b^{-/-}$model at 4-8 months of age was significantly increased (Figure 3B). The length of PTZ-induced seizures in the Epm $2 a^{-/-}$mice at 9-23 months of age was also increased, although no significant differences were observed between old Epm $2 a^{-/-}$or Epm $2 b^{-1-}$ mice and age-matched controls. 

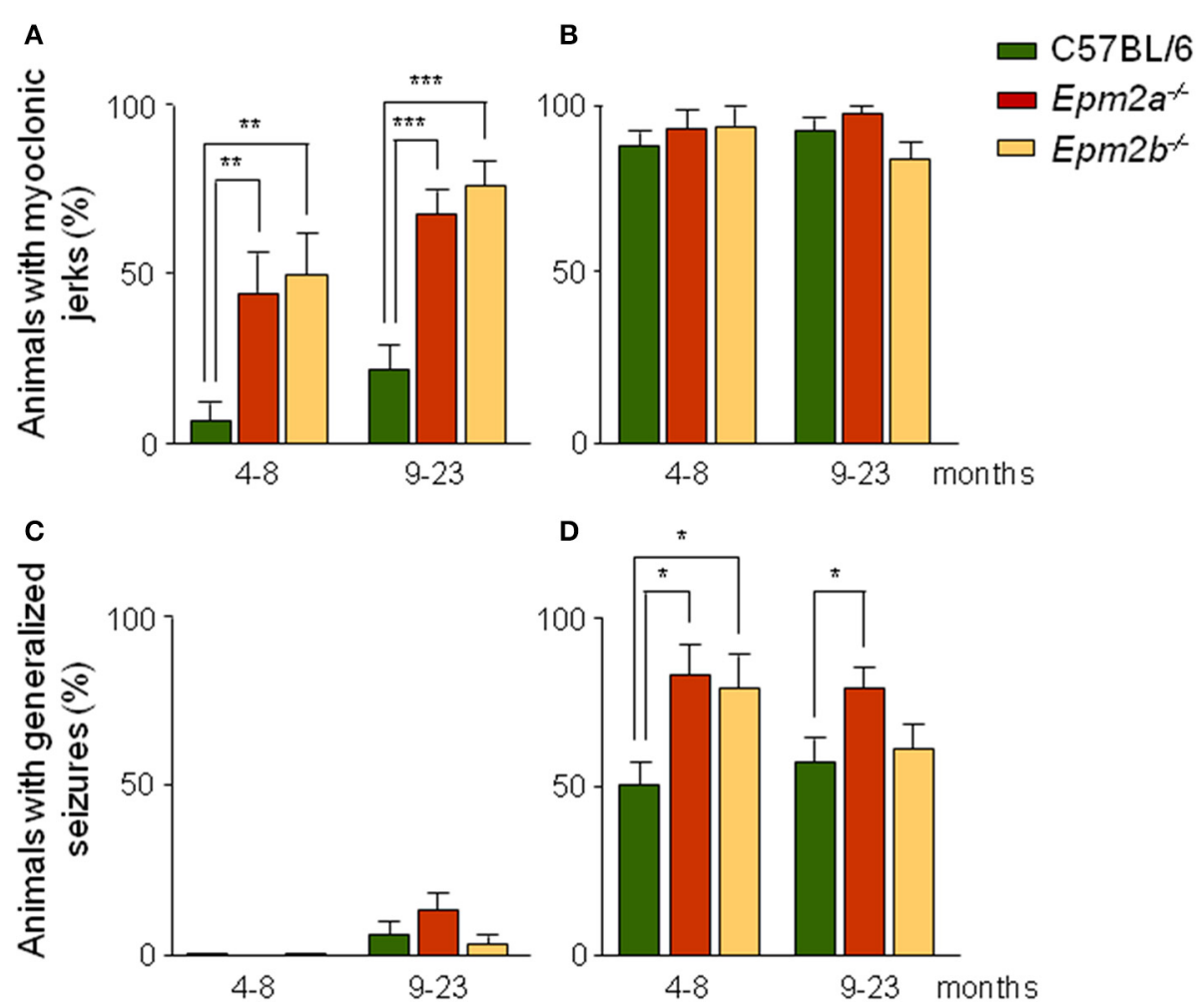

FIGURE 2 | Increased sensitivity of Epm2a-/- and Epm2 $\mathbf{b}^{-/-}$ models to the chemoconvulsant agent PTZ. Two different age groups (4-8 and 9-23 months) of control, Epm2a-/-, and Epm2 $\mathrm{b}^{-/-}$ mice were injected intraperitoneally with $30(\mathbf{A}, \mathbf{C})$ and $50 \mathrm{mg} / \mathrm{kg}$ of
PTZ (B,D) and the percentages of mice showing PTZ-induced myoclonus $(\mathbf{A}, \mathbf{B})$, and generalized seizures $(\mathbf{C}, \mathbf{D})$ were recorded ${ }^{*} p<0.05$; ${ }^{* *} p<0.01$; ${ }^{* * *} p<0.001$, Chi-square $(n=16-36$ mice per group at each PTZ dose).

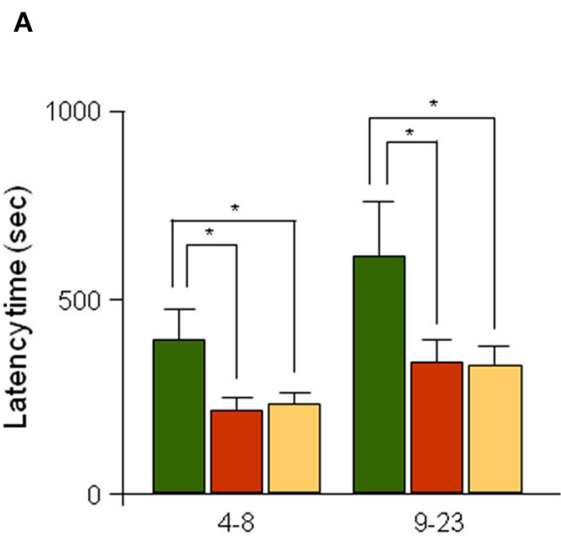

FIGURE 3 | Analysis of seizure latency and length of PTZ-induced generalized seizures in Epm2a $\mathbf{a}^{-/-}$and Epm2 $\boldsymbol{b}^{-/-}$mice. Mice within 2 different age spans (4-8 and 9-23 months) were injected with a convulsive dose of PTZ $(50 \mathrm{mg} / \mathrm{kg})$. (A) The time interval between drug

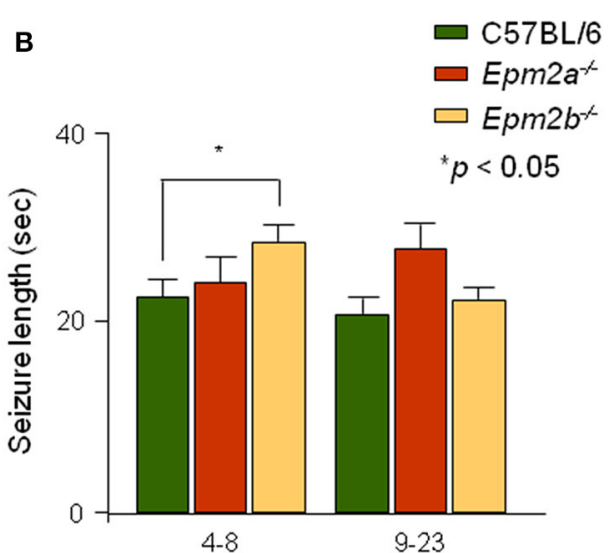

administration and development of generalized tonic-clonic seizures and (B) the seizure length were measured. Data are presented as mean \pm s.e.m. Student's $t$-test was performed for statistical evaluation.

$* p<0.05$ ( $n=12-25)$.
VIDEO-EEG RECORDINGS OF PTZ-INDUCED SEIZURES IN CONTROL, Epm2a $^{-/-}$, AND Epm2b ${ }^{-/-}$MICE AT 16 MONTHS OF AGE

Injections of PTZ at convulsive doses in control mice produced periods of immobility followed by twitching and hyperextension of the limbs, further progressing to generalized tonic-clonic seizures. Intracranial EEG recordings of control, Epm $2 a^{-/-}$, and $E p m 2 b^{-/-}$mice showed different specific patterns of discharges after $50-\mathrm{mg} / \mathrm{kg}$ PTZ injections. Thus, operated control mice 


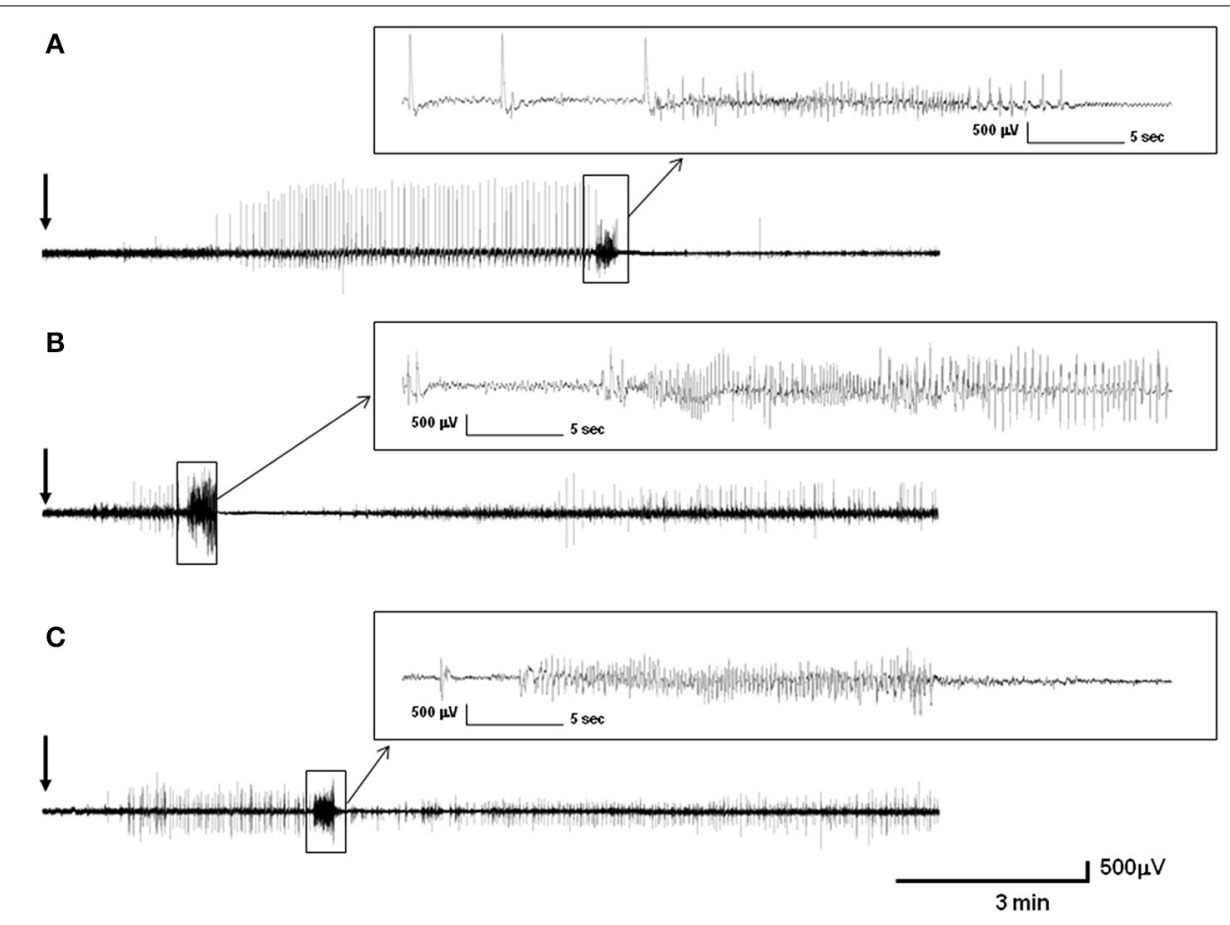

FIGURE 4 | Intracranial representative recording of control, Epm2a-/-, and $E p m 2 b^{-/-}$mice at 16 months of age after injection of $50 \mathrm{mg} / \mathbf{k g}$ pentylenetetrazol (PTZ). (A) Electroencephalographic activity of a control mouse (A), of an Epm2a $a^{-1-}$ mouse (B), and of an $E p m 2 b^{-1-}$ mouse (C). The figure shows the records from monopolar electrodes placed over the left frontal cortex with the reference electrodes implanted posterior to lambda. (representative example is shown in Figure 4A) showed a pattern of discharges starting $1 \mathrm{~min}$ and $19 \mathrm{~s}$ after PTZ injection, with isolated spike and spike-wave discharges alternating with immobility periods during $3 \mathrm{~min}$. At this point, rhythmic isolated spike and spike-wave discharges with muscular jerk correlates were shown over a period of $5 \mathrm{~min}$; these evolved to generalized tonicclonic seizures with low-frequency $(3-6 \mathrm{~Hz})$ spike-wave correlates lasting $22 \mathrm{~s}$. The tonic-clonic seizure began with a tonic phase that was followed by a clonic stage and an after-seizure period of immobility. A representative EEG recording of an Epm $2 a^{-/-}$ mouse after PTZ injection (Figure 4B) displayed a significantly shorter period of time ( $1 \mathrm{~min}$ and $30 \mathrm{~s}$ ) before a brief 1-min initial stage of irregular twitches and myoclonus that correlated with spike and spike-wave complexes and that evolved into a tonic-clonic seizure lasting $29 \mathrm{~s}$. This seizure started with a tonic phase lasting $14 \mathrm{~s}$ with hyperextension of the limbs and was followed by a 4 -s period of clonic stage and a 24 -s period of tonic hind-limb extension. The first $11 \mathrm{~s}$ of this tonic phase presented with spike-wave complex correlates and concluded with a 13-s period of non-neuronal activity. A representative example of EEG recording of an Epm $2 b^{-/-}$mice (Figure 4C) showed a 1-min period of normal activity after PTZ injection, which was followed by myoclonus, muscular jerks, and twitches with EEG correlates of spikes and spike-wave and polyspike-wave complexes over a period of $3 \mathrm{~min}$, preceding a generalized tonic-clonic seizure lasting $22 \mathrm{~s}$. This seizure started with a 3 -s tonic phase with hyperextension of the limbs that was followed by a prominent tonicclonic stage lasting $19 \mathrm{~s}$. The post-seizure period of non-neuronal activity was almost absent in the Epm $2 b^{-/-}$model while postictal discharges with myoclonic correlates started suddenly in this model.

\section{DISCUSSION}

Although a great effort has been devoted to understanding the basis of the formation of Lafora bodies and their relationship to autophagy and alterations in glycogen metabolism (Gentry et al., 2007; Tagliabracci et al., 2008; Aguado et al., 2010; Criado et al., 2012), the relationships between the development of Lafora bodies and the induction of abnormal electroencephalographic activity remain unknown. Lafora disease patients present a distinctive EEG pattern characterized by slowing of background activity with recurrent epileptiform discharges: 3-6 Hz spikes/polyspikes, with or without slow waves (Van Heycop Ten Ham, 1974), similar to that observed in spontaneous seizures developed by Epm2a-1and Epm $2 b^{-/-}$mice (Ganesh et al., 2002; Garcia-Cabrero et al., 2012).

It has been previously reported that the lack of expression of malin protein in a different Epm $2 b^{-/-}$mice model (VallesOrtega et al., 2011) resulted in an increase in the susceptibility to pharmacologically induced hippocampal seizures using the epileptogenic kainic acid. PTZ is a widely used chemical for the induction of generalized epilepsy. It has also been extensively used to test antiepileptic drugs on laboratory animals (Stone, 1970). Although animal models based on PTZ are widely used, the mechanism by which PTZ elicits its action is not very well understood. At the molecular level, a generally 
accepted mechanism of PTZ is non-competitive antagonism of the gamma-aminobutyric acid $\mathrm{GABA}_{\mathrm{A}}$ receptor complex. Additionally, alterations in voltage-dependent calcium and potassium channels and changes in the serotonergic and in the NMDA receptor-mediated glutamate neurotransmission are also generated after PTZ treatment. Treatment with PTZ in mice induces a wide range of responses, from mild convulsions to generalized seizures, all of them as a result of an excess of activation of neurons located in the frontal cortex, the amygdala, the cerebellum, the brainstem, and other regions of the brain.

In this study we have undertaken a systematic evaluation of PTZ-induced seizure responses in Epm $2 a^{-1-}$ and Epm $2 b^{-/-}$ mice. Both models present a significantly increased sensitivity to PTZ that is more noticeable in the presence of myoclonus at subconvulsive doses. Although both Epm $2 a^{-/-}$and Epm $2 b^{-/-}$mice trend to present more myoclonic jerks with age, no statistical differences were found between young and older mice. At convulsive doses, the percentage of Epm $2 a^{-/-}$and Epm $2 b^{-/-}$mice showing generalized seizures was significantly increased, as was seizure length, while the latency time to the appearance of these seizures experienced a statistically significant reduction when compared to age-matched controls. Although old Epm $2 b^{-1-}$ mice trended to be more resistance to PTZ than young animals, no significant differences were found between all aged groups. It has been widely reported that number of Lafora bodies increases with age in brain of Epm $2 a^{-/-}$and Epm $2 b^{-/-}$mice (Ganesh et al., 2002; Criado et al., 2012; Garcia-Cabrero et al., 2012). Moreover, alterations in motor coordination, activity impairment and memory deficits progressively increase with age in both Epm $2 a^{-/-}$and Epm $2 b^{-/-}$mice (Garcia-Cabrero et al., 2012), probably reflecting the increased accumulation of Lafora bodies in multiple brain regions. However, myoclonus and seizures were observed in both mutant mouse strains regardless of age. Thus, it seems likely that development of Lafora bodies does not interfere with the induction of abnormal electroencephalographic activity in our mouse models.

The lack of laforin and malin expression may induce some aberrant activity of neuronal networks, resulting in increased $\mathrm{GABA}_{\mathrm{A}}$ receptor-mediated hyperexcitability. EEG records of PTZ-induced seizures in Epm $2 a^{-/-}$and Epm $2 b^{-/-}$mice showed the presence of characteristic patterns of discharges. Both Epm $2 a^{-/-}$and Epm $2 b^{-/-}$mice display a shorter period of latency with irregular spike and spike-wave discharges before the appearance of the tonic-clonic seizure as compared to agematched controls. Moreover, post-ictal discharges with myoclonic correlates started suddenly in both models. This behavior may reflect the existence of alterations in the GABAergic neurotransmission which could be involved in the generation of epileptic traits.

Overall, our results show that mice lacking expression of either laforin or malin display different degrees of increased $\mathrm{GABA}_{\mathrm{A}}$ receptor-mediated hyperexcitability after administration of the epiletogenic agent PTZ. Our Epm $2 a^{-/-}$and Epm $2 b^{-/-}$models may assist in understanding the way laforin and malin mutations regulate excitotoxic damage and in assaying novel agents aimed at reducing epileptic seizures in this devastating disease.

\section{ACKNOWLEDGMENT}

We thank to Dr. José Luis Sarasa for supervising neuropathological analysis. We also thank Oliver Shaw for editing assistance. This work was supported by grants from the Spanish Ministry of Science and Innovation (SAF2010-18586 to José M. Serratosa), from the Fondo de Investigaciones Sanitarias (PI13/00865) from the Spanish Ministry of Health, and by the Centro de Investigación Biomédica en Red de Enfermedades Raras, CIBERER, Madrid, Spain. Ana M. García-Cabrero and Gentzane Sánchez-Elexpuru were supported by fellowships from the Fundación Conchita Rábago.

\section{REFERENCES}

Acharya, J. N., Satishchandra, P., and Shankar, S. K. (1995). Familial progressive myoclonus epilepsy: clinical and electrophysiologic observations. Epilepsia 36, 429-434. doi: 10.1111/j.1528-1157.1995.tb00482.x

Aguado, C., Sarkar, S., Korolchuk, V. I., Criado, O., Vernia, S., Boya, P., et al. (2010). Laforin, the most common protein mutated in Lafora disease, regulates autophagy. Hum. Mol. Genet. 19, 2867-2876. doi: 10.1093/hmg/ddq190

Berkovic, S. F., Andermann, F., Carpenter, S., and Wolfe, L. S. (1986). Progressive myoclonus epilepsies: specific causes and diagnosis. N. Engl. J. Med. 315, 296-305. doi: 10.1056/NEJM198607313150506

Berkovic, S. F., So, N. K., and Andermann, F. (1991). Progressive myoclonus epilepsies: clinical and neurophysiological diagnosis. J. Clin. Neurophysiol. 8, 261-274. doi: 10.1097/00004691-199107010-00003

Carpenter, S., and Karpati, G. (1981). Ultrastructural findings in Lafora disease. Ann. Neurol. 10, 63-64. doi: 10.1002/ana.410100116

Chan, E. M., Bulman, D. E., Paterson, A. D., Turnbull, J., Andermann, E., Andermann, F., et al. (2003). Genetic mapping of a new Lafora progressive myoclonus epilepsy locus (EPM2B) on 6p22. J. Med. Genet. 40, 671-675. doi: 10.1136/jmg.40.9.671

Chan, E. M., Omer, S., Ahmed, M., Bridges, L. R., Bennett, C., Scherer, S. W., et al. (2004). Progressive myoclonus epilepsy with polyglucosans (Lafora disease): evidence for a third locus. Neurology 63, 565-567. doi: 10.1212/01.WNL.0000133215.65836.03

Cloix, J. F., and Hevor, T. (2009). Epilepsy, regulation of brain energy metabolism and neurotransmission. Curr. Med. Chem. 16, 841-853. doi: $10.2174 / 092986709787549316$

Coimbra, N. C., Castro-Souza, C., Segato, E. N., Nora, J. E., Herrero, C. F., Tedeschi-Filho, W., et al. (2001a). Post-ictal analgesia: involvement of opioid, serotoninergic and cholinergic mechanisms. Brain Res. 888, 314-320. doi: 10.1016/S0006-8993(00)03103-6

Coimbra, N. C., Freitas, R. L., Savoldi, M., Castro-Souza, C., Segato, E. N., Kishi, R., et al. (2001b). Opioid neurotransmission in the post-ictal analgesia: involvement of mu(1)-opioid receptor. Brain Res. 903, 216-221. doi: 10.1016/S0006-8993(01)02366-6

Criado, O., Aguado, C., Gayarre, J., Duran-Trio, L., Garcia-Cabrero, A. M., Vernia, S., et al. (2012). Lafora bodies and neurological defects in malin-deficient mice correlate with impaired autophagy. Hum. Mol. Genet. 21, 1521-1533. doi: $10.1093 / \mathrm{hmg} / \mathrm{ddr} 590$

Depaoli-Roach, A. A., Tagliabracci, V. S., Segvich, D. M., Meyer, C. M., Irimia, J. M., and Roach, P. J. (2010). Genetic depletion of the malin E3 ubiquitin ligase in mice leads to lafora bodies and the accumulation of insoluble laforin. J. Biol. Chem. 285, 25372-25381. doi: 10.1074/jbc.M110.148668

Dudek, F., Clark, S., Williams, P.A., and Grabenstatter, H. L. (2006). "Kainateinduced status epilepticus: a chronic model of acquired epilepsy," in Models of Seizures and Epilepsy, eds A. Pitkänen, P. A. Schwartzkroin, and S. L. Moshé (Burlington, MA: Elsevier Academic Press), 415-432.

Eid, T., Ghosh, A., Wang, Y., Beckstrom, H., Zaveri, H. P., Lee, T. S., et al. (2008). Recurrent seizures and brain pathology after inhibition of glutamine synthetase in the hippocampus in rats. Brain 131, 2061-2070. doi: 10.1093/brain/awn133

Eloqayli, H., Dahl, C. B., Gotestam, K. G., Unsgard, G., Hadidi, H., and Sonnewald, U. (2003). Pentylenetetrazole decreases metabolic glutamate turnover in rat brain. J. Neurochem. 85, 1200-1207. doi: 10.1046/j.1471-4159.2003.01781.x

Erakovic, V., Zupan, G., Varljen, J., Laginja, J., and Simonic, A. (2001). Altered activities of rat brain metabolic enzymes caused by pentylenetetrazol 
kindling and pentylenetetrazol-induced seizures. Epilepsy Res. 43, 165-173. doi: 10.1016/S0920-1211(00)00197-2

Ganesh, S., Delgado-Escueta, A. V., Sakamoto, T., Avila, M. R., Machado-Salas, J., Hoshii, Y., et al. (2002). Targeted disruption of the Epm2a gene causes formation of Lafora inclusion bodies, neurodegeneration, ataxia, myoclonus epilepsy and impaired behavioral response in mice. Hum. Mol. Genet. 11, 1251-1262. doi: $10.1093 / \mathrm{hmg} / 11.11 .1251$

Garcia-Cabrero, A. M., Marinas, A., Guerrero, R., De Cordoba, S. R., Serratosa, J. M., and Sanchez, M. P. (2012). Laforin and malin deletions in mice produce similar neurologic impairments. J. Neuropathol. Exp. Neurol. 71, 413-421. doi: 10.1097/NEN.0b013e318253350f

Gentry, M. S., Dowen, R. H. 3rd., Worby, C. A., Mattoo, S., Ecker, J. R., and Dixon, J. E. (2007). The phosphatase laforin crosses evolutionary boundaries and links carbohydrate metabolism to neuronal disease. J. Cell Biol. 178, 477-488. doi: $10.1083 /$ jcb.200704094

Gentry, M. S., Worby, C. A., and Dixon, J. E. (2005). Insights into Lafora disease: malin is an E3 ubiquitin ligase that ubiquitinates and promotes the degradation of laforin. Proc. Natl. Acad. Sci. U.S.A. 102, 8501-8506. doi: 10.1073/pnas.0503285102

Gomez-Abad, C., Gomez-Garre, P., Gutierrez-Delicado, E., Saygi, S., Michelucci, R., Tassinari, C. A., et al. (2005). Lafora disease due to EPM2B mutations: a clinical and genetic study. Neurology 64, 982-986. doi: 10.1212/01.WNL.00001 54519.10805.F7

Harriman, D. G., Millar, J. H., and Stevenson, A. C. (1955). Progressive familial myoclonic epilepsy in three families: its clinical features and pathological basis. Brain 78, 325-349. doi: 10.1093/brain/78.3.325

Lafora, G. R. (1911). Über das Corkommen amyloider Körperchen im Innern der Ganglienzellen; zugliech Ein zum Studium der amyloiden Substanz im Nervensystem. Virchows Arch. Pathol. Anat. 205, 294-303.

Lafora, G. R., and Glueck, B. (1911). Beitrag zur Histogpathologie der myoklonischen Epilepsie. Z. Gesamte Neurol. Psychiatr. 6, 1-14. doi: 10.1007/BF0 2863929

Löscher, W. (1997). Animal models of intractable epilepsy. Prog. Neurobiol. 53, 239-258. doi: 10.1016/S0301-0082(97)00035-X

Minassian, B. A., Lee, J. R., Herbrick, J. A., Huizenga, J., Soder, S., Mungall, A. J., et al. (1998). Mutations in a gene encoding a novel protein tyrosine phosphatase cause progressive myoclonus epilepsy. Nat. Genet. 20, 171-174. doi: $10.1038 / 2470$

Mitsuno, S., Takahashi, M., Gondo, T., Hoshii, Y., Hanai, N., Ishihara, T., et al. (1999). Immunohistochemical, conventional and immunoelectron microscopical characteristics of periodic acid-Schiff-positive granules in the mouse brain. Acta Neuropathol. 98, 31-38. doi: 10.1007/s00401 0051048

Panagopoulos, N. T., Kazanis, E., Sotiriou, E., Papanastasiou, P., and Matsokis, N. A. (1998). Effect of the pentylenetetrazole (PTZ) induced seizures on the gabaergic system in the mouse brain. Eur. J. Neurosci. 10, 48.

Pitkanen, A., Clark S., Williams P. A., and Moshé, S. L. (2006). Models of Seizures and Epilepsy. Burlington, MA: Elsevier Academic Press.

Reinhard, J. F., and Reinhard, J. F. J. (1977). "Experimental evaluation of anticonvulsants," in Anticonvulsants, ed J. A.Vida (New York, NY: Academic Press), $57-111$.

Roger, J., Genton, P., and Bureau M., and Dravet. C. (1992). "Progressive myoclonus epilepsies in childhood and adolescence," in Epileptic Syndromes in Infancy, Childhood and Adolescence, eds J. Roger, M. Bureau, C. Dravet, F. E. Dreifuss, A. Perret and P. Wolf (London: John Libbey), 381-400.

Sakai, M., Austin, J., Witmer, F., and Trueb, L. (1970). Studies in myoclonus epilepsy (Lafora body form). II. polyglucosans in the systemic deposits of myoclonus epilepsy and in corpora amylacea. Neurology 20, 160-176. doi: 10.1212/WNL.20.2.160

Serratosa, J. M., Gomez-Garre, P., Gallardo, M. E., Anta, B., De Bernabe, D. B., Lindhout, D., et al. (1999). A novel protein tyrosine phosphatase gene is mutated in progressive myoclonus epilepsy of the Lafora type (EPM2). Hum. Mol. Genet. 8, 345-352. doi: 10.1093/hmg/8.2.345
Shitak, R., Sahai, A. K., Hota, D., and Chakrabarti, A. (2006). Anti-seizure efficacy of nimodipine in pentylenetetrazole and kainic acid combined seizure models in mice. Indian J. Physiol. Pharmacol. 50, 265-272.

Singh, S., Sethi, I., Francheschetti, S., Riggio, C., Avanzini, G., Yamakawa, K., et al. (2006). Novel NHLRC1 mutations and genotype-phenotype correlations in patients with Lafora's progressive myoclonic epilepsy. J. Med. Genet. 43, e48. doi: 10.1136/jmg.2005.039479

Singh, S., Suzuki, T., Uchiyama, A., Kumada, S., Moriyama, N., Hirose, S., et al. (2005). Mutations in the NHLRC1 gene are the common cause for Lafora disease in the Japanese population. J. Hum. Genet. 50, 347-352. doi: 10.1007/s10038-005-0263-7

Snead, O. C. 3rd., Banerjee, P. K., Burnham, M., and Hampson, D. (2000). Modulation of absence seizures by the GABA(A) receptor: a critical rolefor metabotropic glutamate receptor 4 (mGluR4). J. Neurosci. 20, 6218-6224.

Stone, W. E. (1970). Convulsant actions of tetrazole derivatives. Pharmacology 3 , 367-370. doi: 10.1159/000136093

Swinyard, E. A., Woodhead, J. H., White, H. S., and Franklin, M. R. (1989). "Experimental selection, quantification, and evaluation of anticonvulsants," in Antiepileptic drugs, eds R. H. Levy, F. E. Dreifuss, R. H. Mattson, B. S. Meldrum, and J. K. Penry (New York, NY: Raven Press), 85-102.

Tagliabracci, V. S., Girard, J. M., Segvich, D., Meyer, C., Turnbull, J., Zhao, X., et al. (2008). Abnormal metabolism of glycogen phosphate as a cause for Lafora disease. J. Biol. Chem. 283, 33816-33825. doi: 10.1074/jbc.M807428200

Turnbull, J., Wang, P., Girard, J. M., Ruggieri, A., Wang, T. J., Draginov, A. G., et al. (2010). Glycogen hyperphosphorylation underlies lafora body formation. Ann Neurol. 68, 925-933. doi: 10.1002/ana.22156

Valles-Ortega, J., Duran, J., Garcia-Rocha, M., Bosch, C., Saez, I., Pujadas, L., et al. (2011). Neurodegeneration and functional impairments associated with glycogen synthase accumulation in a mouse model of Lafora disease. EMBO Mol. Med. 3, 667-681. doi: 10.1002/emmm.201100174

Van Heycop Ten Ham, M. W. (1974). "Lafora disease, a form of progressive myoclonus epilepsy," in The Epilepsies, Handbook of Clinical Neurology, eds. P. J. Vinken and G. W. Bruyn (North-Holland, Amsterdam), 382-422.

Velisek, L. (2006). "Models of chemically-induced acute seizures," in Models of Seizures and Epilepsy, (Burlington, MA: Elsevier Academic Press), 127-152.

Vernadakis, A., and Woodbury, D. M. (1969). The developing animal as a model. Epilepsia 10, 163-178. doi: 10.1111/j.1528-1157.1969.tb03841.x

Woodbury, D. M., Glaser, G. H., Penry, J. K., and Woodbury, D. M. (1980). "Convulsant drugs: mechanism of action," in Advances in Neurology, (New York, NY: Raven Press), 249-303.

Yokoi, S., Austin, J., Witmer, F., and Sakai, M. (1968). Studies in myoclonus epilepsy (Lafora body form). I. isolation and preliminary characterization of Lafora bodies in two cases. Arch. Neurol. 19, 15-33. doi: 10.1001/archneur.1968.00480010033002

Conflict of Interest Statement: The authors declare that the research was conducted in the absence of any commercial or financial relationships that could be construed as a potential conflict of interest.

Received: 13 June 2014; accepted: 27 August 2014; published online: 12 September 2014.

Citation: García-Cabrero AM, Sánchez-Elexpuru G, Serratosa JM and Sánchez MP (2014) Enhanced sensitivity of laforin- and malin-deficient mice to the convulsant agent pentylenetetrazole. Front. Neurosci. 8:291. doi: 10.3389/fnins.2014.00291

This article was submitted to Neurodegeneration, a section of the journal Frontiers in Neuroscience.

Copyright (๑) 2014 García-Cabrero, Sánchez-Elexpuru, Serratosa and Sánchez. This is an open-access article distributed under the terms of the Creative Commons Attribution License (CC BY). The use, distribution or reproduction in other forums is permitted, provided the original author (s) or licensor are credited and that the original publication in this journal is cited, in accordance with accepted academic practice. No use, distribution or reproduction is permitted which does not comply with these terms. 